\title{
Chest injury complicated by shock induced necrosis of the colon
}

\author{
M ASHOUR \\ From the Department of Cardiothoracic Surgery, University Hospital of Wales, Cardiff
}

Many investigators have considered the intestinal vasculature to be a unique and early haemodynamic target in circulatory shock.' Colon necrosis has been reported to be of two types ${ }^{2}$ : type 1 develops spontaneously in older patients as a result of occlusive or non-occlusive vascular disease and occurs mostly in patients with congestive heart failure $^{3}$; type 2 occurs in patients of various ages and appears to be due to a period of documented post-traumatic hypotension; it has been described after multiple injuries and severe lacerations ${ }^{24}$ and in two patients with chest injuries. ${ }^{5}$ The purpose of this report is to describe a case of left chest stabbing leading to shock induced necrosis of the colon.

\section{Case report}

A 55 year old miner was admitted to hospital after being stabbed in the left chest. He was unconscious and shocked with a systolic blood pressure of $60 \mathrm{~mm} \mathrm{Hg}$. He was bleeding from the site of the stab, where the knife was still in place through the left 4 th intercostal space $5 \mathrm{~cm}$ from the sternal margin (fig 1). He was immediately ventilated, a chest tube was inserted, and he was transfused with plasma and blood.

He continued to bleed excessively and his general condition deteriorated so he was transferred to our hospital. On arrival, four hours after the stabbing, he was comatose and not responding to painful stimuli, his pupils were dilated but reactive, and his systolic blood pressure was $40 \mathrm{~mm}$ Hg. A left thoracotomy was immediately performed. The bleeding was coming from the left internal mammary artery and an extensive laceration of the left upper lobe. The divided ends of the internal mammary artery were identified and ligated and a left upper lobectomy was performed. He was ventilated overnight. He was transfused with a total of 30 units of whole blood, 10 units of plasma protein fraction, 6 units of fresh frozen plasma, and 4 litres of dextrose-saline solution. Twelve hours after operation he was extubated. He was well and fully conscious with a normal blood pressure and good urinary output. A chest radiograph showed clear lung fields and a fully expanded left lower lobe.

On the second postoperative day he had abdominal distension without tenderness and bowel sounds were absent. He had a sinus tachycardia of 120 per minute. Abdominal radiographs showed generalised small and large bowel distension. He was treated for paralytic ileus with intravenous

Address for correspondence: Mr M Ashour, FRCS, PO Box 3778, 31481 Dammam, Saudi Arabia.

Accepted 15 February 1985

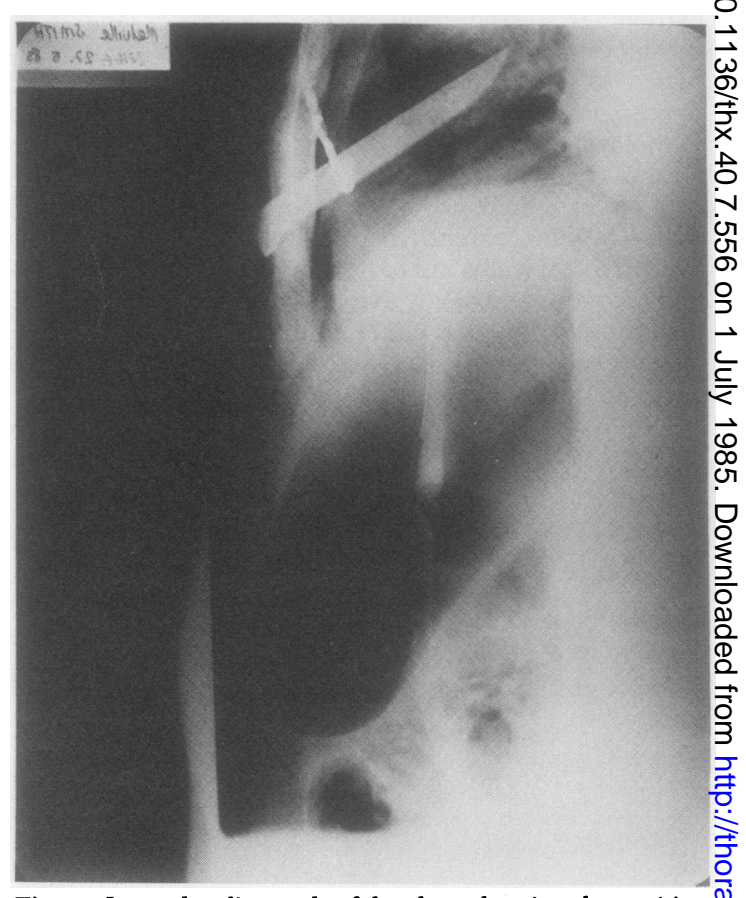

Fig 1 Lateral radiograph of the chest showing the position $\stackrel{x}{2}$ of the knife, the chest drain, and the partially collapsed left $\overline{-}$ lung.

infusions and nasogastric aspiration, having nothing by mouth. By the seventh day the abdomen was more distended and he had bloody diarrhoea. On the 10th day he? had massive abdominal distension with generalised ten- $\rightarrow$ derness. Abdominal radiographs showed dilatation of the colon, which measured $17 \mathrm{~cm}$ in its widest part, and intramural gas had appeared (fig 2). Laparotomy was per-o formed. The entire colon except for the sigmoid loop and rectum was massively dilated, grey, and featureless. The terminal few centimetres of the ileum were also dilated and ${ }^{w}$ grey looking but the rest of the abdominal contents were normal. The inferior mesenteric artery, middle colic arterye and right colic artery were all found to be pulsating nor $-\mathbb{D}$ mally. A subtotal colectomy with fashioning of an ileos? tomy and sigmoid mucous fistula was performed.

After operation the patient made a good recovery. His $-\overline{0}$ tological examination of the excised colon showed multiple areas of ulceration, which penetrated the full thickness of the mucosa. The submucosa looked oedematous with myocytolysis and oedema of the muscularis propria. There 


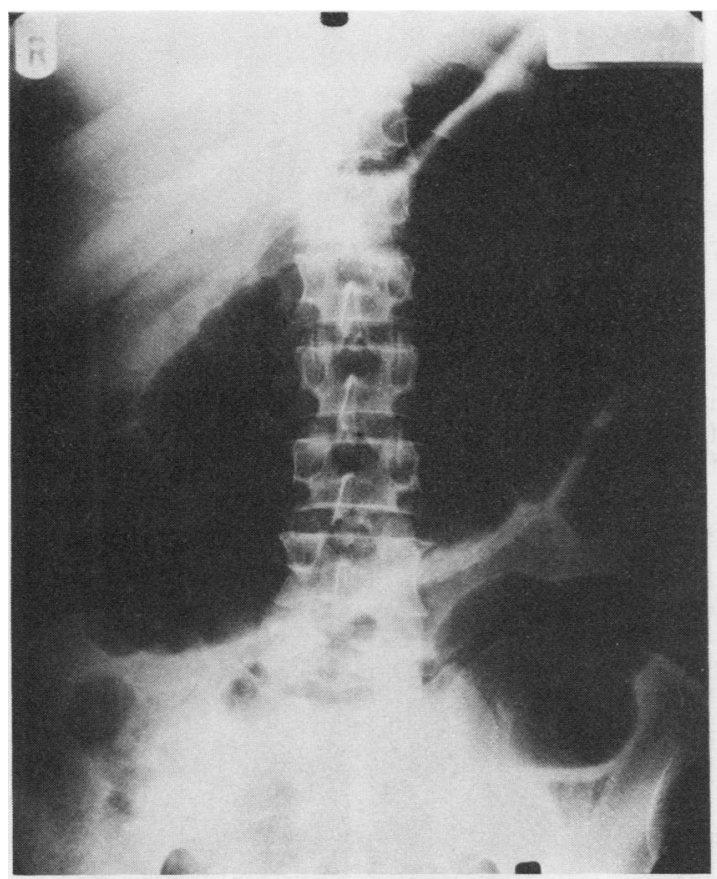

Fig 2 Supine radiograph of the abdomen showing massive dilatation of the colon with irregularity of the mucosa and intramural gas.

were no microscopic abnormalities of the colonic arteries and no thrombi or atherosclerotic patches. Three months later he was admitted electively for ileorectal anastomosis, after which he made an excellent recovery. When seen one year later he was well and symptom free.

\section{Discussion}

Two cases of post-traumatic necrosis of the colon complicating chest injury have been reported by Flynn $e t$ al, both caused by gunshot wounds. ${ }^{5}$ The possible mechanisms thought to cause this form of ischaemia are: $(a)$ a resistance to blood flow through the gut wall due to adrenergic constriction of arteriolar and venous smooth muscles ${ }^{6} ;(b)$ redistribution of blood flow away from the mucosa ${ }^{7}$; and (c) the intestinal countercurrent mechanism, which shunts oxygen away from the tips of the villi, compounding the low flow state and leading to tissue necrosis. ${ }^{8}$ Experimentally, shock induced intestinal ischaemia can be prevented if intestinal segments are perfused intraluminally with oxygenated saline. ${ }^{9}$

It is likely that the shock induced intestinal ischaemia in patients with chest injuries will be compounded by hypoxia due to haemopneumothorax or pulmonary contusion. The biosynthesis of mucin is affected by decreased cellular respiration, exposing the mucosa to the digestive activity of the intestinal pancreatic proteases and leading to the haemorrhagic necrosis and bloody diarrhoea that may occur in these patients. ${ }^{10}$

Interestingly, the eight patients with post-traumatic shock induced necrosis of the colon referred to in this report ${ }^{4}$ were all male. In the two cases described by Sakai et al the sex was not stated. ${ }^{2}$ Males appear to be more susceptible to the effects of trauma and shock than females. Altura found that oestrogenic hormones play a role in the amelioration of an organism's reaction to systemic stress from severe trauma and control the macrophage and peripheral vascular functions. Inbred females have a greater ability to clear particulate matter from the bloodstream than do males of the same strain. Furthermore, females are more resistant to intestinal ischaemia and whole body trauma."

In any patient who has been shocked as a result of chest injury or any other injury and who later develops abdominal distension, with or without tenderness, ischaemia of the bowel should be considered, especially if there has been no history of abdominal injury.

I am most grateful to Mr E Butchart, consultant cardiothoracic surgeon, for his support in the writing of this case report.

\section{References}

1 Ross G. Escape of mesenteric vessels from adrenergic and non-adrenergic vasoconstriction. Am J Physiol 1971;221:1217-22.

2 Sakai L. Keltner R, Kaminski D. Spontaneous and shock associated ischaemic colitis. Am J Surg 1980;140:755-76.

3 Musa BU. Intestinal infarction without mesenteric vascular occlusion. A report of 31 cases. Ann Intern Med 1965;63:783-92.

4 Gully HR, Stewart IP. Ischaemic colitis with perforation in a patient with multiple injuries. Injury 1982;14:100-2.

5 Flynn TC, Rowland BJ, Gilliland M, Ward RE, Fischer RP. Hypotension-induced post-traumatic necrosis of right colon. Am J Surg 1983;146: 715-8.

6 Norris HT, Sumner DS. Distribution of blood flow to the layers of the small bowel in experimental cholera. Gastroenterology 1974;66:973-81.

7 Chou CC, Yu LC, Yu YM. Effects of acute haemorrhage and carotid artery occlusion on compartmental microcirculation in the G I tract. In: Proceedings of the First World Congress for Microcirculation. Toronto: 1975.

8 Haglund U, Hulten N, Ahren E, Lundgren O. Mucosal lesions in the human small intestine in shock. Gut 1975;16:979-84.

9 Ahren C, Haglund U. Mucosal lesions in the small intestine of the cat during low flow. Acta Physiol Scand 1973;88:541-50.

10 Bounous G. Mechanisms of intestinal lesions in shock. Gastroenterology 1975;68:203-4.

11 Altura BM. Sex and estrogen in protection against circulatory stress reactions. Am J Physiol 1976;231:842-7. 